\title{
Modelo matemático para un Vehículo aÉreo no TRIPULADO DE ALA FIJA, USANDO UN ESTIMADOR DE PARÁMETROS "FILTRO DE KALMAN"
}

\author{
Paula A. Ortiz Valencia ${ }^{1}$ \\ Luis Benigno GutiérRez ZeA ${ }^{2}$
}

\section{Resumen}

En este trabajo se plantea un modelo matemático para un Vehículo Aéreo no Tripulado (UAV) que está desarrollando la Universidad Pontificia Bolivariana (UPB) en el grupo de investigación en automática y diseño $(\mathrm{A}+\mathrm{D})$ dentro del macro proyecto "Desarrollo de un vehículo aéreo no tripulado de ala fija para inspección y vigilancia $\left(A U R A^{3}\right)$ ". Para desarrollar el modelo de la aeronave, se siguieron dos pasos: En el primero se obtuvieron las ecuaciones dinámicas que rigen el comportamiento de la aeronave en términos de coeficientes aerodinámicos que dependen de las características de la aeronave en particular. En el segundo paso se encontró el valor de estos parámetros utilizando un filtro extendido de Kalman.

\section{Palabras claves}

UAV, Filtro de Kalman, Coeficientes Aerodinámicos

\footnotetext{
1 Ingeniera en Instrumentación y Control. Magíster en Ingeniería Área Automática. Docente de tiempo completo Instituto Tecnológico Metropolitano. paulaortiz@itm. edu.co

2 Ingeniero electrónico. PhD. Docente Investigador Universidad Pontificia Bolivariana. lbgutier@ieee.org

3 Aura: Nombre dado a la Aeronave por el grupo de investigación A+D

Fecha de recepción: 25 de marzo de 2009

Fecha de aceptación: 18 de mayo de 2009
} 
[76] Modelo matemático para un vehículo aéreo no tripulado de ala fija...

\section{Abstract}

On this work a mathematical model is presented for an UAV that is being developed in UPB (Pontifical Bolivarian University) for the Automatics and Design investigation group (A+D), in the project "Design of a fixed wing unmanned flying vehicle for inspection and vigilance (AURA)". In order to develop the UAV model, two steps were followed: On the first one, the mathematical equations describing aircraft behavior were gotten, in terms of aerodynamical coefficients, depending on particular aircraft characteristics. On the second step, the values of these coefficients were found using an extended Kalman filter.

\section{Keywords}

UAV, Kalman Filter, Aerodynamical Coefficients. 


\section{INTRODUCCIÓN}

El estudio de los vehículos no tripulados (UV de Unmanned Vehicles) se constituye en un área importante de la robótica móvil. Sus aplicaciones van desde la verificación de condiciones climáticas, hasta búsqueda y rescate, pasando por la vigilancia e inspección de territorios y todo tipo de estudios geográficos y orográficos. Lograr la autonomía e inteligencia necesarias para que estos vehículos tomen las decisiones que les permitan llegar al destino prefijado requiere de un sistema de control basado en un modelo matemático confiable del vehículo. La obtención de tal modelo consta de dos partes (Campbell, 2001). La primera, obtener las ecuaciones dinámicas que rigen el comportamiento de la aeronave a partir de la derivación de las fuerzas y momentos que actúan en ella. De esta manera, se tiene la estructura del modelo matemático, en términos de algunos coeficientes aerodinámicos que varían según las características de la aeronave que se desea modelar. Entonces, sigue la segunda parte, obtener el valor de estos coeficientes utilizando alguna técnica de identificación.

Existen pocos reportes sobre la obtención de coeficientes aerodinámicos a partir de datos reales de vuelo. Klein, en su libro "Aircraft System Identification", utiliza métodos de identificación por mínimos cuadrados y mínimos cuadrados recursivos. En el trabajo de Bandu, (1998), se explican los principios aerodinámicos básicos, la estabilidad y las ecuaciones de movimiento de la aeronave. Se describe cómo las fuerzas y momentos aerodinámicos que actúan sobre un avión se crean y cómo pueden modelarse matemáticamente a fin de generar las ecuaciones de movimiento para el vehículo. Luego se muestra cómo estas ecuaciones pueden linearizarse alrededor de una condición de estado estacionario y luego separarse en diferentes coordenadas, de manera que se deriven dos modelos desacoplados para el avión, uno longitudinal y otro lateral. Ambos modelos quedan expresados en términos de derivadas aerodinámicas, ecuaciones de movimiento, de estabilidad y de la dinámica de la aeronave. En el trabajo de Gertjan Looye 
(1999) se presenta una documentación detallada del modelamiento matemático del avión y las posibles perturbaciones a las que se encuentra sometida la aeronave, incluyendo su implementación en Matlab ${ }^{\circledR}$ y Simulink ${ }^{\circledR}$.

Michael Kordt y Helmut Lusebrink (KORDT, 2001) desarrollaron nuevos métodos para la reducción del orden de modelos dinámicos de aviones por medio de ecuaciones diferenciales no lineales

El aporte fundamental de este trabajo consiste en un enfoque generalizado para el modelamiento de una aeronave, de manera que las ecuaciones de fuerza y momento sean aplicables a cualquier vehículo de este tipo. Aunque los coeficientes aerodinámicos son únicos y dependen de las condiciones de vuelo, se pretende que la técnica de identificación usada sirva como referencia para la identificación de modelos de otras aeronaves y que el modelo matemático desarrollado pueda ser utilizado en el diseño de los sistemas de navegación y control que se apliquen sobre estos vehículos.

\section{ECUACIONES DE FUERZA y MOMENTO}

En general, el modelo se expresa por medio de ecuaciones de movimiento, las cuales abarcan tres componentes (Bandu, 1998): el modelo aerodinámico, el sistema de propulsión y el modelo atmosférico. El modelo aerodinámico se explica a continuación:

\subsection{Ecuaciones de fuerza}

Una aeronave de ala fija es un cuerpo elástico, su masa y su densidad cambian con el tiempo y, además tiene partes móviles sujetas a fuerzas aerodinámicas, propulsivas y gravitacionales, sin embargo, se asume lo siguiente para poder modelar el sistema (Stevens, 2003):

- La aeronave es un cuerpo rígido con masa fija y distribución de masa constante

- El aire está en reposo respecto a la tierra 
- La tierra está fija en el espacio inercial

- El vuelo en la atmósfera está cerca a la superficie de la tierra (a escala astronómica), de manera que la superficie terrestre es aproximadamente plana

- La fuerza de gravedad es uniforme

Con estas suposiciones, el movimiento general de la aeronave de ala fija puede ser descrito por la segunda ley de Newton en forma traslacional y rotacional, tal como lo muestran las siguientes ecuaciones:

$$
\begin{aligned}
& F=\frac{d}{d t}(m V) \\
& M=\frac{d}{d t}(I \omega)
\end{aligned}
$$

Donde $F$ es la fuerza, $m V$ es el momento lineal, $m$ es la masa, $V$ es la velocidad traslacional, $M$ es el momento aplicado en el c.g, $I \omega$ es el momento angular aplicado en el c.g, $\omega$ es la velocidad angular e $I$ es la matiz de inercia.

Las ecuaciones (1) y (2) son válidas para un marco de referencia inercial, pero es más conveniente expresar las fuerzas y momentos en el marco del cuerpo (Jones, 2005), así:

$$
V=\left[\begin{array}{c}
u \\
v \\
w
\end{array}\right] \quad M=\left[\begin{array}{c}
M_{x} \\
M_{y} \\
M_{z}
\end{array}\right] \quad \omega=\left[\begin{array}{c}
p \\
q \\
r
\end{array}\right] \quad I=\left[\begin{array}{ccc}
I_{x} & -I_{x y} & -I_{x z} \\
-I_{y x} & I_{y} & -I_{y z} \\
-I_{z x} & -I_{z y} & I z
\end{array}\right]
$$

Ya que la aeronave de ala fija es un cuerpo rígido con simetría relativa en el eje del cuerpo, la matriz de inercia $I$ es simétrica y:

$$
I_{x y}=I_{y x}=I_{y z}=I_{z y}=0
$$

Multiplicando la matriz de inercia $I$ por la velocidad angular $\omega$, se tiene el momento angular aplicado en el c.g $(I \omega)$ : 


$$
I \omega=\left[\begin{array}{c}
I_{x} p-I_{x z} r \\
I_{y} q \\
-I_{x z} p+I_{z} r
\end{array}\right]
$$

La velocidad traslacional $V$ y la velocidad angular $\omega$ representan el movimiento relativo al eje inercial, tal como lo muestra la siguiente ecuación:

$$
\frac{d}{d t}(\square)=\frac{\partial}{\partial t}(\square)+\omega \times(\square)
$$

Reemplazando en la ecuación (6) los valores de $F, V$ y $\omega$, se tiene:

$$
\left[\begin{array}{c}
\dot{u} \\
\dot{v} \\
\dot{w}
\end{array}\right]=\frac{1}{m}\left[\begin{array}{c}
\sum x \\
\sum y z
\end{array}\right]-\left[\begin{array}{c}
p \\
q \\
r
\end{array}\right] \times\left[\begin{array}{l}
u \\
v \\
w
\end{array}\right]
$$

Desarrollando las operaciones indicadas en (7), se tiene:

Donde

$$
\left[\begin{array}{c}
\dot{u} \\
\dot{v} \\
\dot{w}
\end{array}\right]=\left[\begin{array}{l}
(1 / m)\left(\sum x\right)+r v-q w \\
(1 / m)\left(\sum y\right)+p w-r u \\
(1 / m)\left(\sum z\right)+q u-p v
\end{array}\right]
$$

$$
\begin{aligned}
x & =x_{T}+x_{A}+x_{G} \\
\sum y & =y_{T}+y_{A}+y_{G} \\
\sum z & =z_{T}+z_{A}+z_{G}
\end{aligned}
$$




$$
\left[\begin{array}{c}
\dot{u} \\
\dot{v} \\
\dot{w}
\end{array}\right]=\left[\begin{array}{c}
(1 / m)\left(x_{T}+x_{A}+x_{G}\right)+r v-q w \\
(1 / m)\left(y_{T}+y_{A}+y_{G}\right)+p w-r u \\
(1 / m)\left(z_{T}+z_{A}+z_{G}\right)+q u-p v
\end{array}\right]
$$

Donde $\left[\begin{array}{lll}x_{A} & y_{A} & z_{A}\end{array}\right]$ es la fuerza aerodinámica expresada en el marco del vehículo en los ejes $x, y$ y $z,\left[\begin{array}{lll}x_{g} & y_{g} & z_{g}\end{array}\right]$ es la fuerza de gravedad en los ejes $x, y$ y $z$ y $F_{T}=\left[x_{T} y_{T} z_{T}\right]$ es la fuerza de propulsión en el eje $x, y, z$. Asumiendo que el empuje dado por el sistema de propulsión actúa a lo largo del eje x del cuerpo, y a través del centro de gravedad, la fuerza de propulsión resulta tener una sola componente a lo largo del eje x, así:

$$
F_{T}=\left[\begin{array}{l}
T \\
0 \\
0
\end{array}\right]
$$

Donde $T$ es la fuerza de propulsión en el eje x.

Descomponiendo cada una de las fuerzas en la ecuación (10), se tiene:

$$
\left[\begin{array}{c}
\dot{u} \\
\dot{v} \\
\dot{w}
\end{array}\right]=\left[\begin{array}{c}
(1 / m)\left(T+\bar{q} S C_{x}-m g \sin (\theta)\right)+r v-q w \\
(1 / m)\left(\bar{q} S C_{y}+m g \sin (\phi) \cos (\theta)\right)+p w-r u \\
(1 / m)\left(\bar{q} S C_{z}+m g \cos (\phi) \cos (\theta)\right)+q u-p v
\end{array}\right]
$$

\subsection{Ecuaciones de momento}

La ecuación de momento para la aeronave de ala fija en movimiento sería:

$$
M=\frac{d H}{d t}=\dot{H}+\Omega \times H
$$


Donde

$$
\begin{gathered}
H=I \Omega \\
M=\frac{\delta}{\delta t}(I \Omega)+\Omega \times I \Omega \\
\frac{\delta \Omega}{\delta t}=I^{-1}(M-\Omega \times I \Omega) \\
M_{T}=M_{A}+M_{p} \\
M_{A}=\left[\begin{array}{c}
l \\
m \\
n
\end{array}\right]
\end{gathered}
$$

Donde $M$ es el momento de propulsión. La Inversa de la matriz de inercia obtenida de esta ecuación es:

$$
I^{-1}=\frac{1}{I x I z-I x z^{2}}\left[\begin{array}{ccc}
I z & 0 & -I x z \\
0 & I x I z-I x z^{2} / I y & 0 \\
-I x z & 0 & I x
\end{array}\right]
$$

Realizando un cambio de variables $\Gamma=l x l z-l x z^{2}$ se tiene la matriz inversa es:

$$
I^{-1}=\frac{1}{\Gamma}\left[\begin{array}{ccc}
I z & 0 & -I x z \\
0 & \Gamma / I y & 0 \\
-I x z & 0 & I x
\end{array}\right]
$$

Reemplazando la ecuación (20), en la ecuación (16), se tiene:

$$
\left[\begin{array}{c}
\dot{p} \\
\dot{q} \\
\dot{r}
\end{array}\right]=\Gamma\left[\begin{array}{ccc}
I z & 0 & -I x z \\
0 & \Gamma / I y & 0 \\
-I x z & 0 & I x
\end{array}\right] \bullet\left(\left[\begin{array}{c}
l \\
m \\
n
\end{array}\right]+M p-\left[\begin{array}{c}
p \\
q \\
r
\end{array}\right] \times\left[\begin{array}{c}
I x p-I x z r \\
I y q \\
-I x z p+I z r
\end{array}\right]\right)
$$


Resolviendo se tienen las ecuaciones de momento:

$$
\begin{gathered}
\Gamma \dot{p}=I x z(I x-I y+I z) p q-\left[I z(I z-I y)+I x z^{2}\right] q r+I z l+I x z n+M p_{x} \\
\qquad \dot{q}=(I z-I x) p r-I x z\left(p^{2}-r^{2}\right)+m+M p_{y} \\
\Gamma \dot{r}=\left[(I x-I y) I x+I x z^{2}\right] p q-I x z[I x-I y+I z] q r+I x z l+I x n+M p_{z}
\end{gathered}
$$

\subsection{Ecuaciones cinemáticas de rotación}

Las ecuaciones cinemáticas rotacionales relacionan la velocidad de cambio de los ángulos de Euler con las componentes de velocidad angular en el marco del vehículo. Las relaciones se expresan usando la matriz de transformación (Stevens, 2003):

$$
\left[\begin{array}{c}
\dot{\phi} \\
\dot{\theta} \\
\dot{\varphi}
\end{array}\right]=\left[\begin{array}{ccc}
1 & 0 & -\sin \theta \\
0 & \cos \phi & \sin \phi \cos \theta \\
0 & -\sin \phi & \cos \phi \cos \theta
\end{array}\right]^{-1}\left[\begin{array}{l}
p \\
q \\
r
\end{array}\right]
$$

Resolviendo se tiene:

$$
\begin{aligned}
& \dot{\phi}=p+\tan \theta(q \sin \phi+r \cos \phi) \\
& \dot{\theta}=q \cos \theta-r \sin \theta \\
& \dot{\varphi}=q\left(\frac{\sin \phi}{\cos \theta}\right)+r\left(\frac{\cos \phi}{\cos \theta}\right)
\end{aligned}
$$

\subsection{Ecuaciones de navegación}

Las ecuaciones de navegación se escriben expresando el vector de velocidad de la aeronave en el marco de la tierra, comenzando con las componentes en el marco del cuerpo, 


$$
\left[\begin{array}{c}
\square \\
x_{E} \\
\square \\
y_{E} \\
\square \\
z_{E}
\end{array}\right]=\left[\begin{array}{ccc}
C \theta C \varphi & C \theta S \varphi & -S \theta \\
(-C \phi S \varphi+S \phi S \theta C \varphi) & (C \phi C \varphi+S \phi S \theta C \varphi) & S \phi C \theta \\
(S \phi S \varphi+C \phi S \theta C \varphi) & (-S \phi C \varphi+C \phi S \theta S \varphi) & C \varphi C \theta
\end{array}\right]^{T}\left[\begin{array}{c}
u \\
v \\
w
\end{array}\right]
$$

Debe tenerse en cuenta que representa la altitud. Reemplazando se tiene:

$$
h=u \sin \theta-v \cos \theta \sin \phi-w \cos \theta \cos \phi
$$

\section{LineALizACIÓN DEL MODElo}

Las ecuaciones de movimiento vistas hasta ahora pueden simplificarse linealizándolas alrededor de punto de referencia. Cuando la condición de referencia se selecciona como estable, es decir, vuelo nivelado sin sideslip $(\beta)$, las ecuaciones linealizadas se desacoplan en dos conjuntos independientes (Marcos, 2004): uno que describe el movimiento longitudinal en el plano de simetría $(V, \alpha, q, \phi)$, y otro que describe el movimiento lateral por fuera de este plano $(\beta, p, r, \alpha)$.

Las ecuaciones linealizadas han sido utilizadas de manera exitosa y extensiva (Etkin, 1996) en análisis de estabilidad y control, por las siguientes razones:

- Muchos movimientos de vuelo pueden describirse por medio de pequeños cambios de velocidad, desde una condición de referencia

- Los efectos aerodinámicos se pueden describir de manera correcta por medio de funciones lineales de variables de estado y de control

- Los análisis de estabilidad prácticos y el diseño de sistemas de control se basan en modelos dinámicos

De esta manera, las ecuaciones de movimiento se linealizarán aplicando la teoría de pequeña perturbación (Klein, 2006). En el desarrollo de este trabajo, basado en el trabajo de (Klein, 2006), 
se selecciona la condición estable como referencia, es decir, vuelo nivelado sin sideslip. De esta manera se obtienen las siguientes ecuaciones para el modelo longitudinal:

$$
\begin{gathered}
\dot{V}=-\frac{\bar{q}_{o} S}{m}\left(\Delta C_{D_{V}} \frac{\Delta V}{V_{o}}+C_{D \alpha} \Delta \alpha+C_{D q} \frac{q \bar{c}}{2 V_{o}}+C_{D \delta} \Delta \delta\right) \Delta C_{D}-g \cos \gamma_{o}(\Delta \theta-\Delta \alpha)-\frac{T_{o} \operatorname{sen} \alpha_{o}}{m} \Delta \alpha \\
\dot{\alpha}=-\frac{\bar{q}_{o} S}{m V_{o}}\left(\Delta C_{L_{V}} \frac{\Delta V}{V_{o}}+C_{L \alpha} \Delta \alpha+C_{L \dot{\alpha}} \frac{\dot{\alpha} \bar{c}}{2 V_{o}}+C_{L q} \frac{q \bar{c}}{2 V_{o}}+C_{L \delta} \Delta \delta\right)+q-\frac{g \operatorname{sen} \gamma_{o}}{V_{o}}(\Delta \theta-\Delta \alpha)-\frac{T_{o} \cos \alpha_{o}}{m V_{o}} \Delta \alpha \\
\dot{\alpha}=-\frac{\bar{q}_{o} S \bar{c}}{I_{y}}\left(\Delta C_{m_{V}} \frac{\Delta V}{V_{o}}+C_{m \alpha} \Delta \alpha+C_{m \dot{\alpha}} \frac{\dot{\alpha} \bar{c}}{2 V_{o}}+C_{m q} \frac{q \bar{c}}{2 V_{o}}+C_{m \delta} \Delta \delta\right) \\
\dot{\theta}=q
\end{gathered}
$$

$\mathrm{Y}$ se obtienen las siguientes ecuaciones para el modelo lateral:

$$
\begin{gathered}
\dot{\beta}=-\frac{\bar{q}_{o} S}{m V_{o}}\left(\begin{array}{c}
C_{Y_{\beta}} \beta+C_{Y_{p}} \frac{p b}{2 V_{o}} \\
+C_{Y_{r}} \frac{r b}{2 V_{o}}+C_{Y_{\delta}} \delta
\end{array}\right)+p \operatorname{sen} \alpha_{o}-r \cos \alpha_{o}+\frac{g \cos \theta_{o}}{V_{o}} \phi \\
\dot{p}-\frac{I_{x z}}{I_{x}} \dot{r}=-\frac{\bar{q}_{o} S b}{I_{x}}\left(C_{l_{\beta}} \beta+C_{l_{p}} \frac{p b}{2 V_{o}}+C_{l_{r}} \frac{r b}{2 V_{o}}+C_{l_{\delta}} \delta\right) \\
\dot{r}-\frac{I_{x z}}{I_{z}} \dot{p}=-\frac{\bar{q}_{o} S b}{I_{z}}\left(C_{n_{\beta}} \beta+C_{n_{p}} \frac{p b}{2 V_{o}}+C_{n_{r}} \frac{r b}{2 V_{o}}+C_{n_{\delta}} \delta\right) \\
\dot{\phi}=p+\tan \theta_{o} r \\
\dot{\psi}=\sec \theta_{o} r
\end{gathered}
$$

Las variables longitudinales $\Delta V, \Delta a, q$ y $\Delta \theta$ aparecen únicamente en las ecuaciones longitudinales, y las variables laterales $\Delta \beta, p, r, \Delta \phi$ y $\Delta \psi$ aparecen únicamente en las ecuaciones laterales. Adicionalmente, se asume que los controles longitudinales afectan únicamente las fuerzas y momentos longitudinales, y similarmente para los controles laterales. Por esta razón, estos dos conjuntos de ecuaciones están desacoplados y pueden resolverse y analizarse separadamente. 


\section{Estimador dE PARÁMETRos}

\section{Filtro extendido de Kalman}

El filtro de Kalman es un estimador óptimo que permite estimar los parámetros desconocidos de un sistema a partir del modelo matemático del sistema, en el cual se debe tomar medida de uno o más sensores, por lo general estas medias presentan ruido. Speyer, (2001).

Funcionamiento del filtro y ecuaciones. En general, el funcionamiento del filtro se puede describir en tres etapas, a saber, Inicialización, Corrección y Predicción. Primero, se da una estimación del estado inicial del sistema de acuerdo con las condiciones iniciales. Seguidamente, con base en un modelo matemático que se tenga del sistema se hace una predicción del valor que tendrá una determinada variable en la próxima medición. Luego, cuando se mide esta variable con algún instrumento o sensor, la diferencia entre el valor medido y el calculado se utiliza para dar la mejor estimación del valor real de la variable, utilizando para ello un factor de peso (ganancia). La ganancia se calcula con información referente a la precisión de los sensores y de los modelos utilizados, dándole mayor credibilidad a los que sean más precisos. La estimación que hace el filtro se utiliza nuevamente para predecir el valor siguiente de la variable.

A continuación se dan las ecuaciones del filtro de Kalman correspondientes a cada una de las etapas mencionadas, suponiendo que se tiene un sistema representado por el siguiente modelo Speyer, (2001):

$$
\begin{aligned}
& \mathbf{x}_{k}=f\left(\mathbf{x}\left(t_{k-1}\right), \mathbf{u}\left(t_{k-1}\right), t_{k-1}\right)+\mathbf{w}_{k-1} \\
& \mathbf{z}_{k}=h\left(\mathbf{x}\left(t_{k}\right), t_{k}\right)+\mathbf{v}_{k} \\
& \mathbf{w}_{k} \approx N\left(0, \mathbf{Q}_{k}\right) \quad \mathbf{v}_{k} \approx N\left(0, \mathbf{R}_{k}\right)
\end{aligned}
$$


Donde $\mathbf{x}\left(t_{k-1}\right)$ es el vector de estados del sistema en el tiempo $k-1, \mathbf{x}\left(t_{k}\right)$ es el vector de estados del sistema en el tiempo $k, \mathbf{z}_{k}$ son las medidas tomadas por los sensores en el tiempo $k, \mathbf{w}_{k}$ y $\mathbf{v}_{k}$ son ruidos de distribución normal y media 0 , con matrices de covarianza $\mathbf{Q}_{k}$ y $\mathbf{R}_{k}$, respectivamente.

- Inicialización

$$
\begin{aligned}
& \mathbf{x}_{0}^{-}=\mathbf{x}_{0} \\
& \mathbf{P}_{0}^{-}=\operatorname{var}\left[\delta \mathbf{x}_{0}^{-}\right]
\end{aligned}
$$

Donde $\mathbf{x}_{0}$ es la estimación inicial del vector de estados del sistema y $\mathbf{P}_{0}^{-}$es la matriz de covarianza de la estimación.

- Propagación

$$
\begin{gathered}
\mathbf{x}^{-}(t)=f(\mathbf{x}(t-1), \mathbf{u}(t), t) \\
\mathbf{P}_{k}^{-}=\boldsymbol{\Phi}_{k} \mathbf{P} \boldsymbol{\Phi}_{k}^{\mathrm{T}}+\mathbf{Q}_{k} \\
\mathbf{\Phi} \approx \mathbf{I}+\mathbf{F} T \\
\mathbf{F}=\frac{\partial f(x, u)}{\partial x}
\end{gathered}
$$

Donde, $\mathbf{F}$ es la Jacobiana de $f, \Phi_{k}$ es la matriz de propagación de estados, que corresponde a la discretización de $\mathbf{F}$ y $\mathbf{Q}_{k}$ es la matriz de covarianza del error en el modelo.

- Actualización

$$
\begin{aligned}
& \mathbf{K}_{k}=\mathbf{P}_{k}^{-} \mathbf{H}_{k}^{\mathrm{T}}\left[\mathbf{R}_{k}+\mathbf{H}_{k} \mathbf{P}_{k}^{-} \mathbf{H}_{k}^{\mathrm{T}}\right]^{-1} \\
& \mathbf{y}_{k}=h\left(\mathbf{x}\left(t_{k}\right), t_{k}\right) \\
& \mathbf{x}_{k}^{+}=\mathbf{x}_{k}^{-}+\mathbf{K}_{k}\left[\mathbf{z}_{k}-\mathbf{y}_{k}\right] \\
& \mathbf{P}_{k}^{+}=\left[\mathbf{I}-\mathbf{K}_{k} \mathbf{H}_{k}\right] \mathbf{P}_{k}^{-}
\end{aligned}
$$

Donde $\mathbf{H}=\frac{\partial h(x, u)}{\partial x}, \mathbf{K}_{k}$ es la ganancia del filtro y $\mathbf{R}_{k}$ es la matriz de covarianza del error en las medidas. 


\section{Aplicación del Filtiro de Kalman}

En esta sección se explica la forma como se estimaron los parámetros de los coeficientes aerodinámicos de la aeronave de ala fija.

\subsection{Pasos para la estimación de los parámetros}

Para la aplicación de las técnicas de identificación se deben tener en cuenta los siguientes:

- Se debe tomar datos de vuelo, ya sean a partir de una prueba real o de una simulación. A continuación se presentan las variables que deben medirse son:

Velocidad lineal $(u, v, w)$, Velocidad angular $(p, q, r)$, Aceleración $\left(a_{x}, a_{y}, a_{z}\right)$, Velocidad del motor $(\Omega)$, Ángulos de Euler ( $\left.\phi, \theta, \psi\right)$, Altitud (h).

- El modelo se divide en dos, un modelo longitudinal con el cual se estiman los coeficientes $C_{L}, C_{D}$ y $C_{m}$ y un modelo lateral con el cual se estiman los coeficientes $C_{y}, C_{l}$ y $C_{n}$.

- Del modelo linealizado se determinan las variables que afectan la estabilidad lateral $\beta, \frac{b}{2 V} p, \frac{b}{2 V}$ y las variables que se necesita manipular $\delta_{a}$ y $\delta_{r}$ y las variables que afectan la estabilidad longitudinal $\alpha, \frac{b}{2 V} q, \dot{\alpha}$, y las variables que se necesita manipular $\delta_{f} \mathrm{y} \delta_{e}$.

Para determinar los coeficientes aerodinámicos, sería necesario hacer una serie de pruebas de vuelo, en las cuales se tomaran mediciones de ciertas variables. Dado que en este proyecto no se tomaron datos reales de vuelo, lo que se hizo fue aplicar un programa desarrollado en la UPB por los docentes Luis Benigno Gutiérrez y Omar Hazbon para obtener los coeficientes aerodinámicos en función de la geometría de la aeronave. Se tomó una base de datos para diferentes condiciones de vuelo y se simularon varias pruebas en dicho programa obteniéndose los datos necesarios para la identificación. Para simular el efecto de los sensores reales, a estos datos obtenidos se le sumó un ruido blanco. 
- Se obtienen los parámetros y se calculan los coeficientes aerodinámicos usando MATLAB.

\subsection{Pruebas de vuelo simuladas para la identificación}

Se realizó en MATLAB la simulación de vuelo de una aeronave, con el objetivo de tomar datos que permitieran la identificación de los parámetros requeridos. De esta manera, se simuló un vuelo en crucero, con una velocidad de viento constante, y se registraron datos sobre altitud, actitud, velocidad lineal y angular. A esta información se le agregó un ruido blanco, con el fin de simular el ruido añadido por los sensores que medirían estas variables. Esta información ruidosa, es la que utilizan los programas de identificación para estimar los parámetros aerodinámicos de la aeronave.

\subsection{Estimación de los parámetros usando el Filtro extendido de Kalman}

Para la aplicación de este método, se divide el sistema en dos partes, una lateral y otra longitudinal.

A continuación se explica el procedimiento para el desarrollo del filtro tanto para el modelo lateral como para el longitudinal. El Filtro de Kalman se implementó en una función de Matlab $^{\circledR}$.

Aplicación del filtro de Kalman al modelo lateral. Para identificar los parámetros del modelo lateral usando el filtro de Kalman, se siguen los pasos a continuación:

- Se define la matriz de estados

$$
x=\left[\begin{array}{l}
X \\
\Theta
\end{array}\right]
$$

Donde son los parámetros desconocidos a ser estimados por el filtro de Kalman y es el vector de estados:

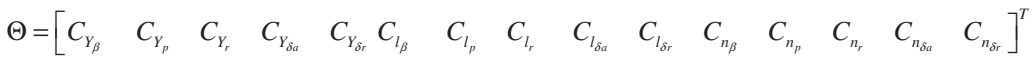

$$
\begin{aligned}
& X=\left[\begin{array}{lllll}
\beta & p & r & \phi & \varphi
\end{array}\right]^{T}
\end{aligned}
$$


- Modelo del sistema.

Habiendo definido el vector de estados, el paso siguiente para la aplicación del filtro de Kalman es obtener un modelo del sistema, el cual ya se obtuvo en la sección 3 .

- Linealización del modelo.

Debido a que se tiene un modelo no lineal para el sistema, es necesario linealizarlo con el fin de aplicar el filtro extendido de Kalman. La linealización consiste principalmente en la obtención de dos Jacobianas y, a partir de las funciones y del sistema:

$$
A=\frac{\partial f(x, t, u)}{\partial x}, \quad H=\frac{\partial h(x, t, u)}{\partial x}
$$

Donde $\dot{x}=f(x, t, u) \quad$ y $\quad y=h(x, t, u)$

- Obtención de las medidas.

Se deben obtener los valores de las variables que serían medidas por los sensores. En este caso no se tienen sensores reales, de manera que se toma la información de las pruebas de simulación, y se les añade ruido blanco. El vector de variables medidas sería:

$$
y=\left[\begin{array}{lllllll}
\beta & p & r & \phi & a_{y} & \dot{p} & \dot{r}
\end{array}\right]
$$

Del vector de medidas $\beta, p, r$ y $\phi$ son variables de estado, $\mathrm{y}$ son las velocidades angulares en los ejes $x$ y $z$, y se calcula a partir de la siguiente ecuación:

$$
a_{y}=\frac{\bar{q} S}{m g}\left(C_{Y_{\beta}} \beta+C_{Y_{p}} \frac{p b}{2 V}+C_{Y_{r}} \frac{r b}{2 V}+C_{Y_{\delta_{a}}}+C_{Y_{\delta_{r}}}\right)+g \operatorname{sen} \phi \cos \theta
$$

Aplicación del filtro de Kalman al modelo longitudinal. Para identificar los parámetros del modelo longitudinal usando el filtro de Kalman, se siguen los pasos a continuación:

- Se define la matriz de estados:

$$
x=\left[\begin{array}{l}
X \\
\Theta
\end{array}\right]
$$


Donde $\Theta$ son los parámetros desconocidos a ser estimados por el filtro de Kalman y $X$ es el vector de estados:

$$
\begin{aligned}
& \Theta=\left[\begin{array}{llllllllll}
C_{D_{0}} & C_{D_{v}} & C_{D_{\alpha}} & C_{D_{\dot{\alpha}}} & C_{D_{q}} & C_{D_{\delta f}} & C_{D_{\delta e}} & C_{L_{0}} & C_{L_{v}} & C_{L_{\alpha}}
\end{array}\right. \\
& \left.\begin{array}{lllllllllll}
C_{L_{\dot{\alpha}}} & C_{L_{q}} & C_{L_{\delta f}} & C_{L_{\delta e}} & C_{m_{0}} & C_{m_{V}} C_{m_{\alpha}} & C_{m_{\dot{\alpha}}} & C_{m_{q}} & C_{m_{\delta f}} & C_{m_{\delta e}}
\end{array}\right]^{T} \\
& X=\left[\begin{array}{llll}
V & \alpha & q & \theta
\end{array}\right]^{T}
\end{aligned}
$$

- Modelo del sistema

Habiendo definido el vector de estados, el paso siguiente para la aplicación del filtro de Kalman es obtener un modelo del sistema, el cual ya se obtuvo en la sección 4 .

- Linealización del modelo

La linealización consiste en la obtención de dos Jacobianas $A$ y $H$, a partir de las funciones $f$ y $h$ del sistema, tal como se mostró para el modelo lateral.

- Obtención de las medidas

El vector de variables medidas sería:

$$
y=\left[\begin{array}{lllllll}
V & \alpha & q & \theta & a_{x} & a_{z} & \dot{q}
\end{array}\right]
$$

Donde $V, \alpha, q$ y $\theta$ son variables de estado, $\dot{q}$ es la velocidad angular en el eje $y, a_{x}$ y $a_{z}$ se calculan a partir de la siguiente ecuación:

$$
\begin{gathered}
a_{x}=(-\bar{q} * S / m) * C_{D}-g * \sin (\theta)+(1 / m) * x_{T} \\
a_{z}=(-\bar{q} * S / m) * C_{L}+g * \cos (\phi) * \cos (\theta)
\end{gathered}
$$

Verificación del método. De la Fig. 1 a la Fig. 6 se muestran las gráficas de la estimación de los parámetros $C_{n}, C_{l}$ y $C_{y}, C_{D}, C_{L}$ y $C_{m}$ usando el filtro extendido de Kalman. Los errores obtenidos se hallaron con la variable estimada y la variable real, la cual fue obtenida a partir de la simulación realizada y del modelo matemático. 

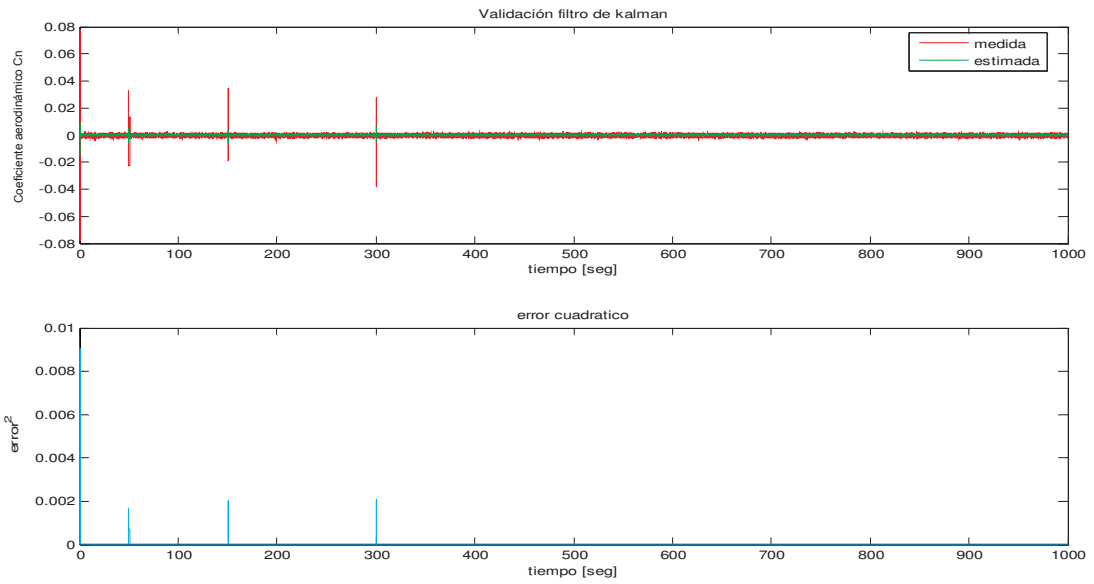

Figura 1. Gráfica del coeficiente de momento estimado,

EN COMPARACIÓN CON EL VALOR REAL DEL PARÁMETRO.

ABAJO SE MUESTRA LA GRÁFICA DEL ERROR EN LA ESTIMACIÓN.
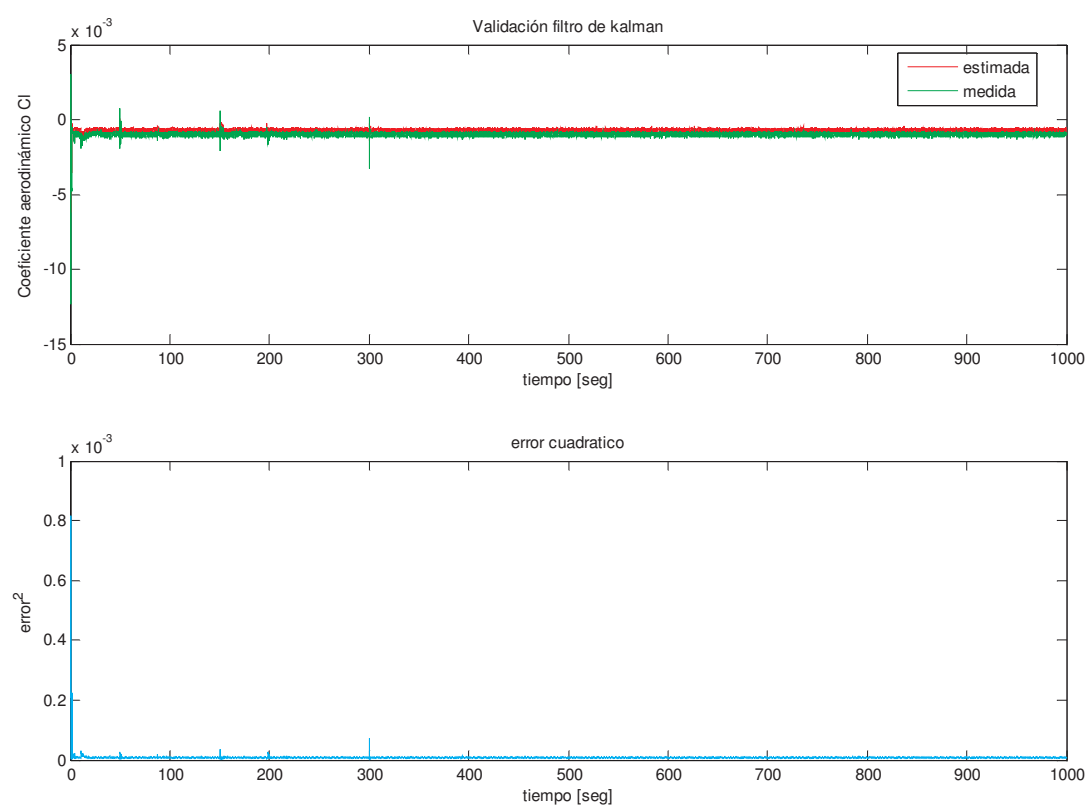

Figura 2. Gráfica del coeficiente de momento estimado,

EN COMPARACIÓN CON EL VALOR REAL DEL PARÁMETRO.

ABAJO SE MUESTRA LA GRÁFICA DEL ERROR EN LA ESTIMACIÓN. 

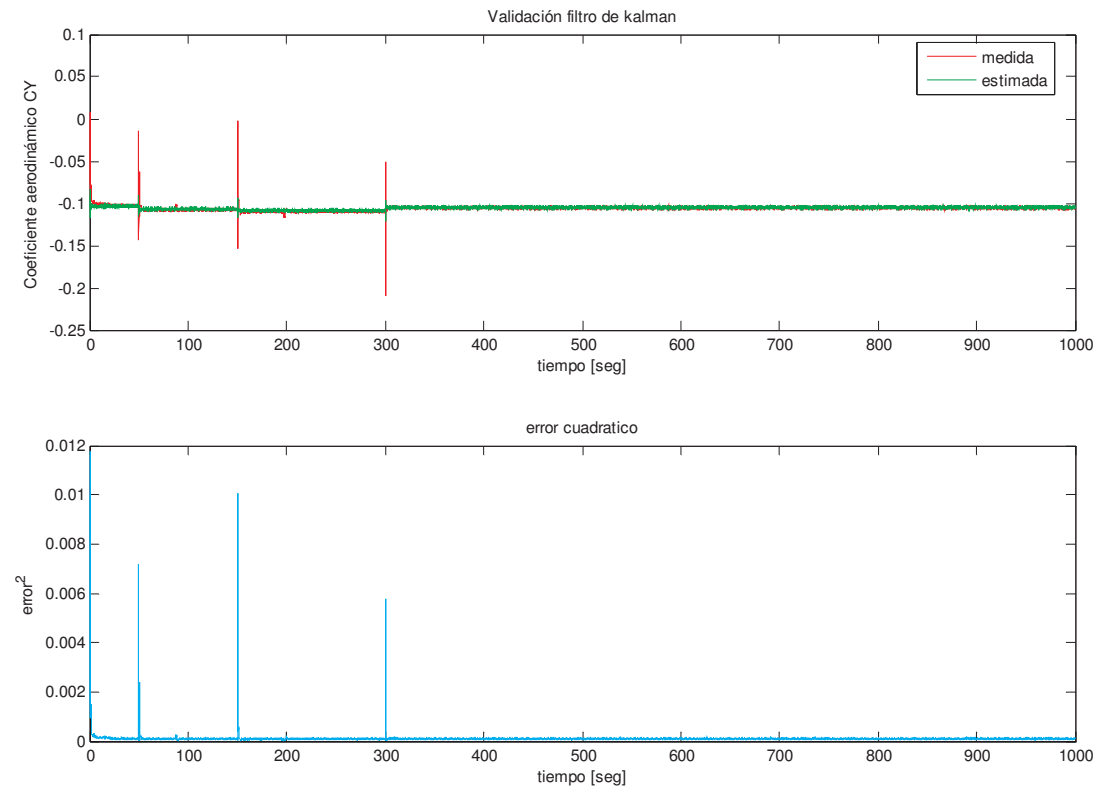

Figura 3. GRÁfica del COEFICIENTE de FUeRZA EStimado, en COMPARACIÓN CON EL VALOR REAL DEL PARÁmETRO. ABAJO SE MUESTRA LA GRÁFICA DEL ERROR EN LA ESTIMACIÓN.
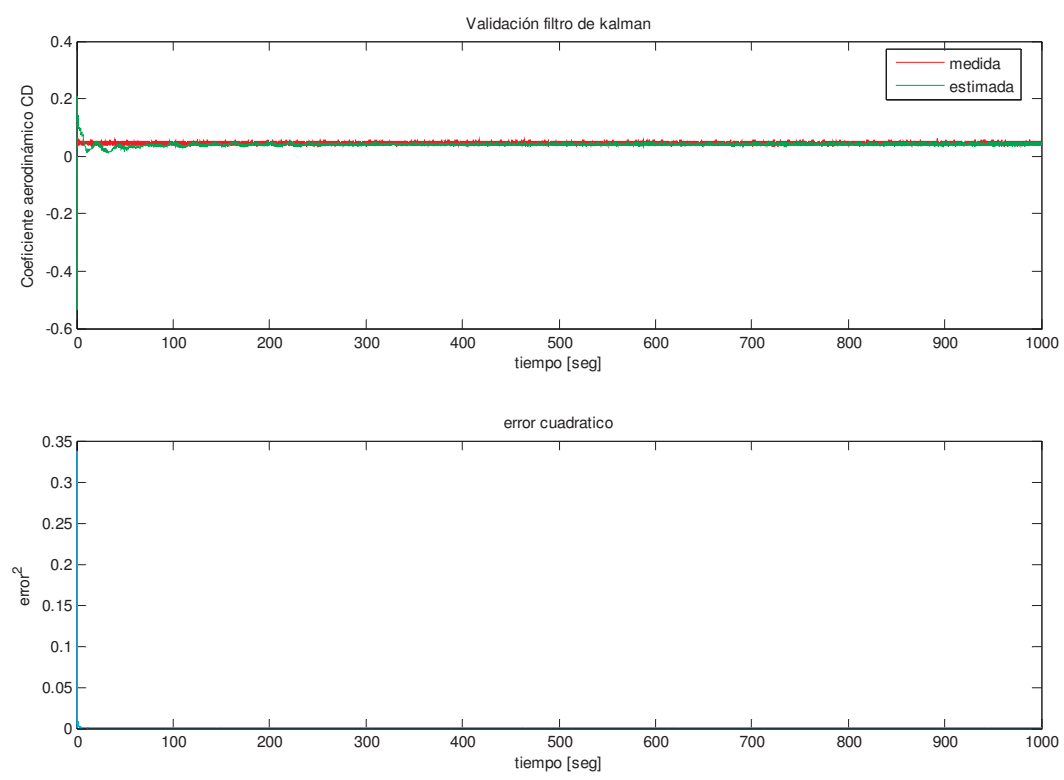

Figura 4. Gráfica del COEFICIENTE de FUeRZA Estimado, EN COMPARACIÓN CON EL VALOR MEDIDO DEL PARÁMETRO. ABAJO SE MUESTRA LA GRÁFICA DEL ERROR EN LA ESTIMACIÓN. 
[94] Modelo matemático para un vehículo aéreo no tripulado de ala fija...
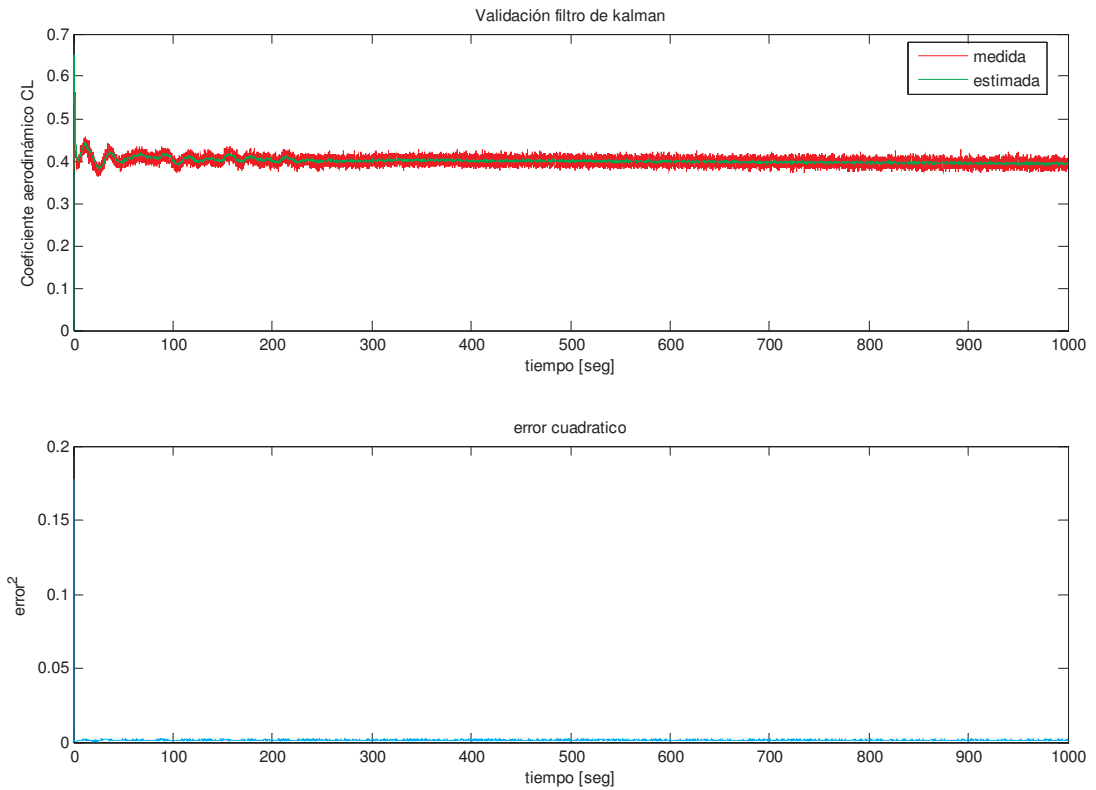

Figura 5. Gráfica del Coeficiente de fuerza estimado, en COMPARACIÓN CON EL VALOR MEdido del parámetro. Abajo SE MUESTRA LA GRÁfica del ERROR EN LA ESTIMACIÓN.
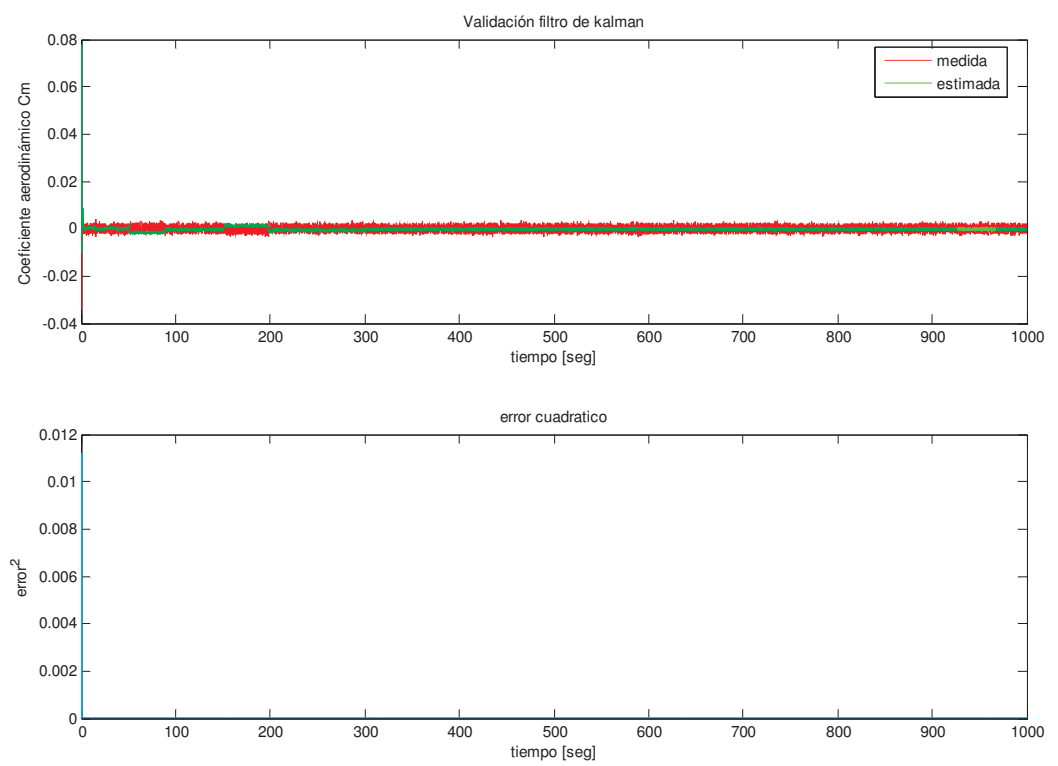

FiguRA 6. GRÁficA DEL COEFICIENTE dE MOMENTO ESTIMADO, EN COMPARACIÓN CON EL VALOR

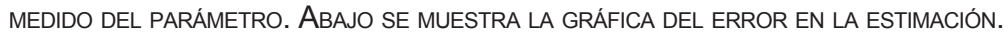




\section{Análisis de RESUltados}

El filtro de Kalman se implementó usando funciones en Matlab, tanto para el sistema lateral como para el sistema longitudinal, para ello fue necesario sintonizar la matrices de covarianza $\mathrm{Q}$ y $R$, de manera que el resultado de la estimación hecha por el filtro de Kalman converja hacia el valor real de la variable.

En la Tabla 1 se muestran los errores promedio y errores cuadráticos medios obtenidos en la estimación de los parámetros, para obtener los valores de $C_{D} C_{L} C_{Y} C_{l} C_{m} C_{n}$ y compararlos con los arrojados por el filtro de Kalman fue necesario desarrollar un programa en Matlab el cual se encargaba de cargar los datos obtenidos en la simulación y a partir del modelo matemático estimar los coeficientes, estos parámetros son los que se asumieron como reales, los datos estimados fueron los calculados con el filtro de Kalman, para ello esta prueba fue realizada off-line, una vez comprobado que los coeficientes encontrados son confiables se puede realizar una estimación on-line, y conocer el modelo matemático en cualquier condición de vuelo.

De los resultados obtenidos se puede observar que el filtro de Kalman no solo es un buen estimador de los parámetros desconocidos, sino que filtra el ruido de la señal proveniente de los sensores.

TABLA 1. ERRORES PROMEDIO Y CUADRÁTICO MEDIO

\begin{tabular}{cccccc}
\hline Coeficiente & $\begin{array}{c}\text { Error } \\
\text { promedio }\end{array}$ & $\begin{array}{c}\text { Error } \\
\text { cuadrático } \\
\text { medio }\end{array}$ & Coeficiente & $\begin{array}{c}\text { Error } \\
\text { promedio }\end{array}$ & $\begin{array}{c}\text { Error } \\
\text { cuadrático } \\
\text { medio }\end{array}$ \\
$C_{D}$ & 0.000112 & 0.0005 & $C_{l}$ & $3,015 \mathrm{e}-4$ & $2,0015 \mathrm{e}-4$ \\
$C_{Y}$ & 0.000104 & 0.0007 & $C_{m}$ & 0.00015 & 0.0002 \\
$C_{L}$ & 0.000393 & 0.0042 & $C_{n}$ & 0.000135 & 0.00045 \\
\hline
\end{tabular}




\section{Conclusiones}

- Al desarrollar el modelo aerodinámico del UAV, obteniendo sus fuerzas y momentos, se puede concluir que las ecuaciones dinámicas y estáticas que rigen su movimiento, son ecuaciones generales que describen el comportamiento de cualquier aeronave de ala fija, sin embargo, éstas dependen de coeficientes aerodinámicos y de propulsión, que son característicos de la arquitectura de cada vehículo, y del tipo de motor y hélice que lo componen

- A partir de los resultados obtenidos en simulación con el filtro de Kalman se puede concluir que éste es capaz de estimar los coeficientes aerodinámicos a partir de datos de vuelo reales, a partir de un modelo matemático desacoplado en un sistema lateral y otro longitudinal

- El filtro de Kalman es ideal para trabajar en tiempo real puesto que sólo requiere los resultados de la última estimación y como ventaja adicional, recibe toda la información que se le pueda proporcionar, de manera que incluso integrar información de otros sensores es una tarea fácil

- La estimación de los coeficientes aerodinámicos con métodos off-line sólo arroja resultados precisos cuando se trabaja en un punto cercano al punto de operación, de lo contrario los errores pueden llegar a ser inaceptables. Esto se puede evitar trabajando con métodos de identificación on-line

- Debido a que el filtro de Kalman depende de la información estadística sobre las medidas de los sensores, cuando desee utilizarse este algoritmo, será necesario cambiar los valores de las matrices Q y R de acuerdo con la dinámica de error de los sensores a utilizar. Esta tarea puede ser dispendiosa ya que en la práctica no se tendría conocimiento del valor real de las variables a estimar

- De los resultados del Filtro de Kalman, puede concluirse que éste obtuvo excelentes resultados, ya que logró estimar bien los parámetros y filtrar el ruido generado por los sensores 


\section{Referencias}

Bandu Pamadi "Performance, Stability, Dynamics, and Control of Airplanes", Second Edition. AIAA Educational Series, New York, 1998.

Campbell, Mark E. and Brunke, Shelby (2001). "Nonlinear Estimation of Aircraft Models for On-line Control Customization". Aerospace Conference, 2001, IEEE Proceedings. Volume 2, Issue, 2001. Page(s):2/621 $2 / 628$.

ETKIN, B., and Reid, L.DD., Dynamics of flight- stability and control. New York: Wiley, 3rd ed,1996. 720 p.

Evans, Jennifer Gabriel Elkaim, Sherman Lo, Dr, Bradfor Parkinson, Stanford University. (2001) "System Identification of an Autonomous Aircraft using GPS".

GERTJAN, Looye and Samir Bennani. Description and analysis of the Research Civil Aircraft Model (RCAM). En Control Engineering Practice. Francia: Vol. 8, no 5 (May. 2000), 750p.

Iliff, Kenneth W. (2002). "Parameter Estimation for Flight Vehicles" AIAA Journal of Guidance and Control, vol. 12 (5) pp. 609-622.

Klein, Vladislav y Morelli, Eugene A. (2006). "Aircraft System Identification”. Ed. AIAA education series, Virginia Polytecnic Institute and State University, Blacksburg, 484p.

Kordt, Michael and Lusebrink, Helmut. (2001). "Nonlinear Order Reduction of Structural Dynamic Aircraft Models". Aerospace Science and Technology, $5(1)$, pp. $55-68$.

Lyashevskiy, Sergey and Chen, Yavbin. (1996). "Nonlinear Identification of Aircraft". Proceedings of the 1996 IEEE lntemational Conference on Control Applications Dearborn, MI September 15-18. pp. 327 - 331.

MARCOS, Andres and Balas, Gary J. Development of Linear-ParameterVarying Models for Aircraft En: Journal of Guidance, Control and Dynamics. George T. Schmidt Massachusetts .Vol. 27, No. 2, (Mar. Abr. 2004), $350 \mathrm{p}$.

Mueller, Joseph B., Paluszek, Michael A. and ZHAO, Yiyuan. (2004) "Development of an Aerodynamic Model and Control Law Design for a High Altitude Airship". AIAA Unmanned Unlimited Conference, Chicago, Sep. 2004. 
SPEYER, Jason L. and Cruest, Edwin Z. Aircraft and Stability Derivative Estimation Using the Modified-Gain Extended Kalman Filter. En: AIAA Journal of Guidance and Control, Vol. 10 no.3, (may. 200); 280p

Stevens, Brian 1. (2003) "Aircraft control and simulation". John Wiley, 2a Ed.New Jersey, Canada, 2003.664 pp. 